\title{
La historia de Ai Fen, Li Wenliang y Zhang Jixian en los albores de la pandemia COVID-19
}

\section{The story of Ai Fen, Li Wenliang and Zhang Jixian at the beginning of COVID-19 pandemic}

Pamo-Reyna P. La historia de Ai Fen, Li Wenliang y Zhang Jixian en los albores de la pandemia COVID-19. Rev Soc Peru Med Interna. 2020;33(3): 10I-106.

https://doi.org/10.36393/spmi.v33i3.546

\begin{abstract}
RESUMEN
La figura del médico chino LiWenliang ha calado en la opinión pública como el primero en alertar a sus colegas sobre la aparición de una nueva enfermedad desconocida con características de epidemia y que por ello fue sancionado por las autoridades chinas. Sin embrago, hay otras dos historias detrás de Li Wenliang: Ai Fen, la médico que realmente fue la que alertó a los demás sobre la naciente epidemia y de Zhanag Jixian, quien oficialmente fue la primera médico que alertó sobre el nacimiento del COVID-I9.
\end{abstract}

Palabras claves: Li Wenliang, Ai Fen, Zhang Jixian, historia, COVID-19, coronavirus, pandemia, China.

\section{ABSTRACT}

The figure of the Chinese doctor LiWenliang has permeated public opinion as the first to alert his colleagues about the appearance of a new unknown disease with epidemic characteristics and for that reason he was sanctioned by the Chinese authorities. However, there are two other stories behind LiWenliang:Ai Fen, the doctor who really was the one who alerted others about the nascent epidemic and Zhang Jixian, who was officially the first doctor who alerted about the birth of COVID-19. Keywords: Li Wenliang, Ai Fen, Zhang Jixian, history, COVID-19, coronavirus, pandemic, China.

El 7 de febrero pasado, una noticia causó la indignación no solo del pueblo chino sino, también, del mundo entero. Había muerto el médico chino Li Wenliang, víctima del COVID-19, la nueva enfermedad por coronavirus SARSCov-2 y causante de la pandemia actual, y de quién se dijo fue el primero en alertar sobre dicha enfermedad mortal, que las autoridades lo reprimieron y lo acusaron de "difundir rumores".(1) Los medios de información hicieron eco de esta noticia y, de inmediato, resaltaron la figura de $\mathrm{Li}$ Wenlinag considerándolo, merecidamente, un mártir de la

Médico internista. Departamento de Medicina, Hospital Nacional Arzobispo Loayza. Facultad de Medicina, Universidad Peruana Cayetano Heredia, Lima, Perú. medicina. Pero, detrás de Li Wenliang había otras historias. La historia de Li Wenliang nos remite a los días primigenios en que apareció la actual pandemia. Los científicos chinos vienen intentando mapear el patrón de transmisión del primer paciente con COVID-19. Todo apunta a que el primer infectado, la primera persona que se contagió de coronavirus fue un hombre de 55 años de edad, a mediados de noviembre del 2019, que vivía en Wuhan, provincia de Hubei. ${ }^{1}$ A partir de ese supuesto primer caso, cada día se fue encontrando de uno a cinco casos nuevos en los diversos hospitales de Wuhan, tal que el 20 de diciembre había 60 casos y a fines del año 2019 se contaba con 266 casos.(2)

\section{Ai Fen y su silbato}

El primer caso de una desconocida infección pulmonar recibido en el Hospital Central de Wuhan data del 16 de diciembre y procedía del Departamento de Emergencias del Hospital Nanjing Road. ${ }^{2}$ El paciente tenía fiebre alta y no respondía al tratamiento habitual de una neumonía. La

I Wuhan, ciudad capital de la provincia de Hubei, en la parte central de China, con un área de $8500 \mathrm{Km}^{2}$ y poco más de II millones habitantes.

2 La provincia de Hubei tiene 995 hospitales, de los cuales 551 son hospitales generales y 279 hospitales especializados. https://www. statista.com/statistics/ 1094979/china-number-of-healthcareinstitutions-in-hubei-province-type/ 
tomografía reveló extenso compromiso de los pulmones. El día 22, el paciente fue trasladado al departamento de neumología, se realizó una fibrobroncoscopia y se tomó líquido de lavado broncoalveolar que fue enviado para secuenciamiento de alto rendimiento (HTS). ${ }^{3}$

En los días siguientes, Ai Fen, directora del Departamento de Emergencia del Hospital Central de Wuhan, recibió, verbalmente, el reporte de "Coronavirus" en dicho paciente. Y, también, se enteró que el paciente trabajaba en el mercado de pescados y mariscos local. ${ }^{4}$

Ai Fen se había graduado de médico en 1997, en la Universidad Médica de Tongji, y había trabajado en el Departamento de Medicina Cardiovascular del Hospital Central de Wuhan. Era directora del Departamento de Emergencias desde el 2010.

El 27 de diciembre, otro paciente vino del Hospital Nanjing Road. Era sobrino de un médico de la Emergencia de Wuhan. Tenía 40 años y ninguna enfermedad subyacente. Había estado hospitalizado diez días y sin mejoría alguna, ingresó con insuficiencia respiratoria. También se le hizo fibrobroncoscopía y el líquido de lavado broncoalveolar fue enviado para HTS.

Simultáneamente, los médicos de otros hospitales empezaron a reportar casos de la extraña neumonía a los centros de control de enfermedades locales de Wuhan. Pero, en China, los informes tienen que pasar primero por la burocracia del Partido Comunista local. Se corrió la noticia de que había un brote de neumonía el cual llegó a los funcionarios del control de enfermedades en Beijing, después que los rumores y los documentos filtrados comenzaron a propagarse online. El Centro Nacional para el Control de Enfermedades evitó señalar en los anuncios que Wuhan lo había notificado, en lugar de aceptar que tenía conocimiento del brote. Las autoridades locales se cubrieron sobre cuándo y cómo le dijeron a Beijing. Los documentos filtrados fueron dos directivas internas del gobierno de Wuhan, que surgieron online el 30 de diciembre, posiblemente publicados por médicos preocupados. Las directivas, marcadas como "urgentes", ordenaron a los hospitales que enviaran a la comisión de salud de la ciudad toda información sobre los casos de la misteriosa neumonía, mejorar el tratamiento de los pacientes y evitar la infección en los hospitales.(3)

Al mismo tiempo, los médicos alertaron a sus colegas sobre el brote de la desconocida neumonía en los chats de grupos privados en las redes sociales. En efecto, el 30 de diciembre, la doctora Ai Fen recibió el informe de laboratorio del paciente con síntomas de una neumonía que no respondía a los tratamientos usuales. Dicho informe

3 Secuenciamiento de alto rendimiento (high-throughput sequencing, HTS) es una técnica que permite secuenciar cientos de millones de moléculas de ADN de manera paralela y a la vez.

4 El mercado mayorista de pescados y mariscos del Sur de China (Huanan, en chino) de Wuhan es un mercado mojado de la ciudad de Wuhan. Está ubicado en el distrito urbano de Jianghan, cerca de la estación de tren de Hankou. En él, además de especies marinas se comercia todo tipo de animales silvestres y exóticos para consumo humano. decía: "Coronavirus $\underline{S A R S}$, Pseudomonas aeruginosa, 46 tipos de bacterias de colonización respiratorias/orales". La mención de SARS la conmocionó. Hizo un círculo sobre la palabra $S A R S$, tomó una foto al informe con su smartphone y la envió a un ex compañero de universidad a través de WeChat. ${ }^{5}$ La imagen comenzó a circular en el ámbito médico. Fua así que este mensaje le llegó a Li Wenliang, médico oftalmólogo del mismo hospital y a otros siete colegas. Li envió el siguiente mensaje, según Caixin, grupo de noticias chino, a otros colegas y compañeros de estudios médicos: "Ha sido confirmada una nueva infección por coronavirus. Informen a todas sus familias y parientes estar atentos". ${ }^{1}$

Ai Fen, graduada en 1997, debió de conocer lo que fue la epidemia de síndrome respiratorio agudo grave de 20022004, SARS (Severe Acute Respiratory Syndrome), causada por el coronavirus SARS-CoV. El brote se identificó por primera vez en Foshán, Guangdong, al sur de China, en noviembre de 2002, se extendió a 29 países de todo el mundo y se tuvo unos 8100 casos confirmados con una letalidad del $11 \%$.(4)

Ai Fen había cumplido con informar al centro de salud comunitaria y al centro de control de enfermedades infecciosas. Esa misma noche del 30 de diciembre, la doctora Fen recibió una llamada de sus jefes. No debía enviar información a otros colegas porque esto podría generar pánico.(5)

Los funcionarios de la Comisión Nacional de Salud dijeron que habían ordenado a Wuhan que emitiera su primer anuncio oficial sobre el brote el 31 de diciembre. Ese día, el gobierno también informó a la oficina de la Organización Mundial de la Salud (OMS; $W H O$, por sus siglas en inglés) en Beijing.

El $1^{\circ}$ de enero, un supervisor del hospital le llamó la atención a Ai Fen por "difundir rumores" y por haber creado desconcierto con sus revelaciones, acusándola de ser una delatora. Para entonces, ya había unas 200 personas afectadas en su hospital. En ese mismo día, se cerró el mercado de pescados y mariscos, sindicado inicialmente por los médicos y las autoridades como el probable epicentro de la infección. Pero, los enfermos continuaron llegando en los días siguientes.

El 3 de enero, Li Wenliang había sido convocado y amonestado por las autoridades del Buró de Seguridad Pública, le hicieron firmar un documento en el cual aceptaba haber realizado informaciones falsas que alteraban el orden público.

Las autoridades indicaron que la enfermedad sería controlable y que no había transmisión de humano a humano. Esto hizo que todo el personal de salud siguiera atendiendo a los enfermos como si se tratara de una enfermedad común; esto es, sin protección.

Una semana después. una enfermera se hospitalizó con un cuadro similar a la desconocida neumonía pero la

5 WeChat es una aplicación usada por la mayoría de los chinos, es mucho más versátil que WhatsApp, es un híbrido entre aplicación, marketplace, medio de pago y casi un sistema operativo. 
dirección del hospital decidió cambiar el diagnóstico de "neumonía viral" a "infección generalizada". Sobre el resto del personal del hospital recayó la orden que "estaba prohibido compartir fotos o cualquier otro mensaje sobre el nuevo virus".

El 12 de enero, las autoridades policiales ordenaron a los médicos que los reportes de enfermedades infecciosas deberían ser remitidos a una instancia superior del distrito. El 13 de enero, Wang Wenyong, quien dirige el control de enfermedades infecciosas en el Centro de Control de Enfermedades del distrito de Jianghan, de Wuhan, dijo a los médicos del Hospital Central de Wuhan que alteraran los informes de sospecha de coronavirus y que reportaran que los pacientes sufrían otras enfermedades. En este día, Tailandia reportó el primer caso de la nueva enfermedad fuera de China, en una mujer que había estado en Wuhan. En los días siguientes, numerosos países del Asia reportaron casos de la inusual neumonía.

En cuanto al informe de Ai Fen a sus superiores sobre lo que estaba sucediendo, le respondieron que la Comisión Sanitaria de la ciudad había ordenado no dar información sobre el posible virus para no crear pánico entre la población. La misma dirigencia del hospital también recordó al personal que estaba prohibida la publicación de informaciones sobre la desconocida enfermedad.

E1 21 de enero, un día después de que los funcionarios chinos confirmaran finalmente que había transmisión de humano a humano del virus, el número de residentes enfermos que llegaban a la sala de emergencias del Hospital Central de Wuhan ya había alcanzado los 1523 en un día, el triple de lo habitual.

Ante el creciente número de afectados y de muertos, el 22 de enero, las autoridades pusieron en cuarentena a Wuhan, la provincia de Hubei y toda China, sucesivamente. Un hecho que ayudó a que lo que se consideró un brote se convirtiera en epidemia, y como amenaza creciente, fue que en esta época del año suele ocurrir desplazamientos de las gentes que vacacionan para visitar a sus familias en las diversas provincias. Porque, precisamente, este era el Año de la Rata de Metal, según el horóscopo oriental, que va del 25 de enero hasta el 11 de febrero del 2021. El Año Nuevo chino comprende celebraciones y la gente suele viajar de las ciudades a sus lugares de origen para las festividades, $o$, simplemente, hacer turismo interno y externo.

El 30 de enero de 2020, se contaban más de 9700 casos confirmados en China y 106 casos confirmados en otros 19 países; y, ese mismo día, la OMS declaró que se trataba de una urgencia de salud pública de alcance internacional.

\section{Vindicación póstuma de Li Wenliang}

En los primeros días de febrero, Li Wenliang enfermó de la nueva enfermedad y tuvo que ser internado en cuidados intensivos. El 7 de febrero, Li Wenliang falleció a consecuencia del COVID-19. En cierto modo, Li había sido el "chivo expiatorio" que las autoridades chinas tomaron para amedrentar a los demás médicos para que no se atrevieran a divulgar información sobre la nueva enfermedad que se comportaba con características de epidemia.

Li Wenlinag nació el 16 de octubre de 1986, en Beizhen, China. Estudió medicina en la Universidad de Wuhan, trabajó en Xiamen, ciudad portuaria, el sudeste de China y, desde el 2014, trabajaba como oftalmólogo en el Hospital Central de Wuhan. Le sobrevivieron sus padres, quienes se habían recuperado del COVID-19, un hijo y su esposa gestante de su segundo hijo.(6)

Casi simultáneamente, con Li Wenlinag fallecieron otros tres médicos de 29, 55 y 57 años de edad. Para entonces, los trabajadores de salud afectados se contaban por algunos miles. Llamó la atención que muriera mucha gente joven entre los trabajadores de salud.(7) Esto último fue explicable por la alta exposición a ambientes con gran carga viral ya que la población con real riesgo de enfermar gravemente fue la de los adultos mayores y los que eran portadores de comorbilidad (diabetes mellitus, obesidad, hipertensión arterial, etc.).

El 11 de febrero, la OMS denominó a la nueva enfermedad como COVID-19 (Coronavirus Disease 2019).(8)

A mediados de febrero se corrió la noticia de que el nuevo coronavirus habría sido creado y escapado de un laboratorio. Esto llevó a diversas especulaciones que involucró a varios científicos y a las más altas autoridades norteamericanas.(9) Lo que contribuía a este rumor era la existencia del Instituto de Virología de Wuhan, situado en las colinas cercanas a la ciudad. Este instituto alberga el Centro de Cultivo de Virus, el banco de virus más importante de Asia, donde se preservan más de 1500 variedades y desde el 2015 contiene el primer laboratorio de Asia de alta seguridad, capaz de manejar patógenos de clase 4 (BSL-4), es decir que se transmiten de persona a persona. El instituto había recibido el nuevo virus el 30 de diciembre, determinó su genoma viral el 2 de enero e informó a la OMS el 11 de enero. $(10,11)$

Un mes después de la muerte de Li Wenliang, la prensa demandaba a las autoridades chinas sobre el supuesto cargo de difundir rumores con que Li Wenlinag y sus colegas habían sido acusados por las autoridades locales.(12)

Luego de extenderse por el planeta, el 6 de marzo se confirmó el primer caso de COVID-19 en nuestro país; y la estadística puede ser seguida en la página oficial del Gobierno Peruano.(13)

El 10 de marzo, coincidente con la primera visita que hiciera el presidente chino, Xi Jinping, a Wuhan, la revista china Ren $W u$ publicó una entrevista que le hicieron a Ai Fen. En sus declaraciones sobre cómo ocurrieron los hechos, Ai Fen criticó a las autoridades del hospital por borrar las primeras advertencias del brote. La entrevista de Ai Fen fue eliminada rápidamente de las redes sociales chinas. Fue en esta entrevista en que ella negó ser una soplona y que ella sólo dio el silbato. ${ }^{6}$ La revista Ren $W u$ borró el artículo y Ai Fen no pudo ser contactada por teléfono. Por fortuna, los internautas guardaron el artículo y remitieron

6 "I am not a whistleblower," Ai told Ren Wu."I am the one who provided the whistle." 
a distintas redes sociales y medios las capturas de pantalla de la entrevista. Para burlar la censura, hicieron versiones escritas parcialmente con emojis, en código morse y en pinyin, sistema romanizado del mandarín.(14)

El 11 de marzo, la OMS anunció que el, inicialmente, brote epidémico de COVID-19 originado en China se ha había convertido en una pandemia. Para entonces, el COVID-19 se había extendido al sudeste asiático y Medio Oriente para alcanzar a Europa como epicentro, con más de 20000 casos confirmados y más de 1000 muertes.(15)

Ante el clamor porque se haga justicia con la memoria de Li Wenliang, en China y en el resto del mundo, el Buró de Seguridad Pública de Wuhan emitió una disculpa a la familia de Li Wenliang por su "reprimenda inapropiada"; y, después anunció que el jefe de la policía de Wuhan recibió un demérito y un oficial de policía recibió una amonestación. El diario oficial del Comité Central del Partido Comunista de China, People's Daily, hizo pública por diversos medios la disculpa. Esta y las medidas disciplinarias, junto con la revocación de la reprimenda a Li Wenliang, fueron dadas a conocer luego de una investigación por el Comité Central Disciplinario dentro de la estación policial responsable de la amonestación a Li Wenliang.(16)

En los seis meses de pandemia por COVID-19 han surgido sucesivas hipótesis sobre la naturaleza artificial del coronavirus causante. Una hipótesis reciente de investigadores de Harvard sugiere que el brote pudo haber empezado en agosto del 2019, basándose en el incremento del tránsito automovilístico alrededor de los hospitales de Wuhan y de la mayor búsqueda por Internet de enfermedades con las entradas de 'diarrea' y 'tos'.(17) Lo concreto hasta ahora es que tenemos a un coronavirus como causante de una pandemia que en seis meses ha matado a más de medio millón de personas en el mundo.

\section{Zhang Jixian y la historia oficial china}

En la mañana del 26 de diciembre de 2019, una pareja de adultos mayores fue derivada al Hospital de Hubei por presentar fiebre y tos. Las tomografías computarizadas de tórax mostraron hallazgos completamente diferentes de las neumonías comunes.(18)

Zhang Jixian, directora del Departamento de Medicina Respiratoria y de Cuidados Críticos, Hospital Provincial de Hubei de Medicina Tradicional China y Occidental Integrada, convocó al hijo de los pacientes para un examen. Este era asintomático pero la tomografía mostró que los pulmones tenían los mismos hallazgos. En ese día, un comerciante del mercado de pescados y mariscos de Huanan fue admitido con fiebre, tos y con similares imágenes pulmonares.?

Los estudios descartaron las habituales infecciones pulmonares pero Zhang Jixian sospechó que se trataba de algo diferente. Así, el 27 de diciembre, informó la situación de las cuatro personas al decano de negocios Xia Wenguang y a las autoridades inmediatas del hospital Estas informa

7 El Hospital Provincial de Hubei de Medicina Tradicional China y Occidental Integrada es uno de los dos hospitales de tercer nivel más cercanos al mercado de mariscos.

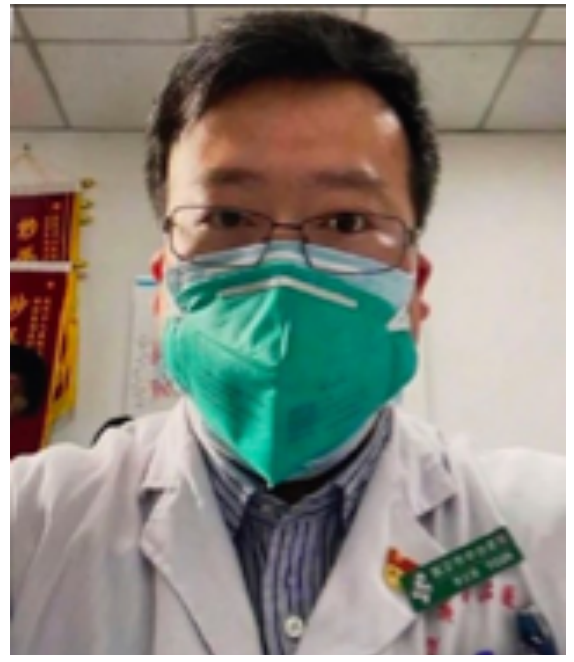

Figura 2. Selfie del Dr. LiWenliang.

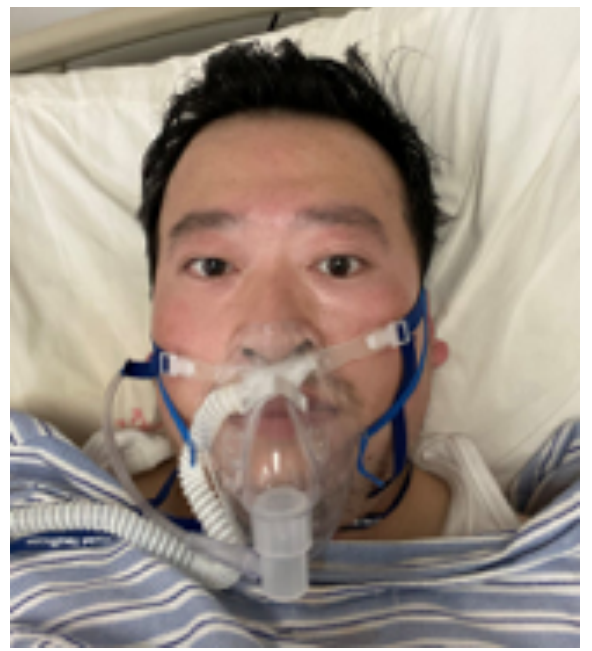

Figura 3. Dr. LiWenliang en UCl, 03-02-2020 (Gan en Fund/Reuters).

ron de inmediato al Centro de Control de Enfermedades del Distrito Jianghan.

E1 28 y 29 de diciembre, ingresaron otros tres pacientes, que también provenían del mercado de pescados y mariscos, con iguales características clínicas y de imágenes que los primeros.

A la una de la tarde del 29 de diciembre, el decano Xia Wenguang convocó a diez expertos del Departamento de Medicina Respiratoria, Oficina de Pacientes Hospitalarios, Cardiovascular, UCI, Radiología, Farmacia, Laboratorio Clínico, Infección y Asuntos Médicos. Revisaron minuciosamente cada caso. También revisaron otros dos casos similares, del Hospital Tongji y el Hospital Xiehe, y con el antecedente de provenir del mercado de pescados y mariscos. El vicepresidente Xia Wenguang informó de inmediato al Departamento de Control de Enfermedades de la Comisión de Salud Provincial y Municipal.

El domingo 29 de diciembre el Departamento de Control y Prevención de Enfermedades de la Comisión de Salud Provincial y Municipal respondió rápidamente y convocó a los CDC de Wuhan, el Hospital Jinyintan 


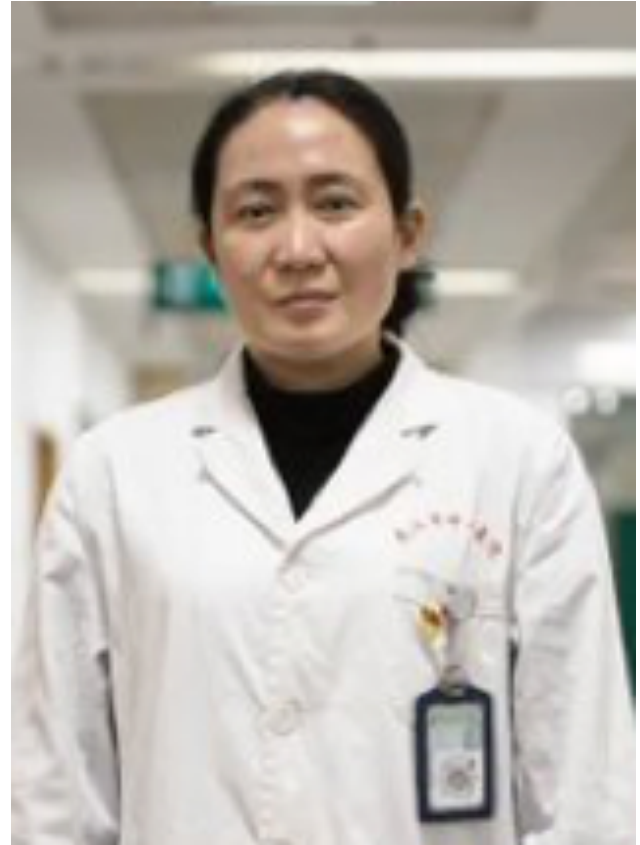

Figura I. Dra.Ai Fen, Hospital de Wuhan, China

y los CDC del Distrito Jianghan al hospital de Hubei para iniciar las investigaciones epidemiológicas. Esa noche, Huang Chaolin, vicepresidente del Hospital Wuhan Jinyintan, y Wu Wenjuan, director de la UCI, acudieron al Hospital Hubei de Medicina Tradicional China y Occidental Integrado y revisaron a los pacientes, excepto al tercer caso que desistió de acudir al hospital. ${ }^{8}$ Zhang Jixian separó un lugar relativamente independiente de otras áreas en la sala respiratoria para aislamiento con nueve camas, en el Hospital Wuhan Jinyitan, donde fueron hospitalizados los primeros casos. la Clínica Respiratoria de Zhang Jixian continuó recibiendo pacientes similares y para el 31 de diciembre las nueve camas de aislamiento eran insuficientes.

Por otro lado, Zhang Jixian solicitó a todo el personal médico del área respiratoria que usara máscaras y ordenó la adquisición de ropa de trabajo a ser usada debajo de la vestimenta convencional de médicos y enfermeras. El hospital aprobó el uso de máscaras protectoras N95 para sus departamentos y recién exigiría el uso de los equipos de protección personal cuando en la tarde del lunes 20 de enero del 2020, el científico Zhong Nanshan declaró que la neumonía causada por el nuevo coronavirus puede transmitirse de persona a persona. ${ }^{9}(19)$

Para el 31 de diciembre, el número de pacientes ambulatorios en el Departamento Respiratorio del Hospital Provincial

8 El Hospital Wuhan Jinyintan es un hospital público que depende del Comité Municipal de Salud de Wuhan y es donde se trata a todos los casos de emergencia de Hubei y Wuhan.

9 El neumólogo Zhong Nanshan lideró el equipo de investigación que descubrió el Síndrome Respiratorio Agudo Severo (SARS) en el 2003. A los 83 años de edad, acudió a Wuhan para estudiar la nueva neumonía por coronavirus.

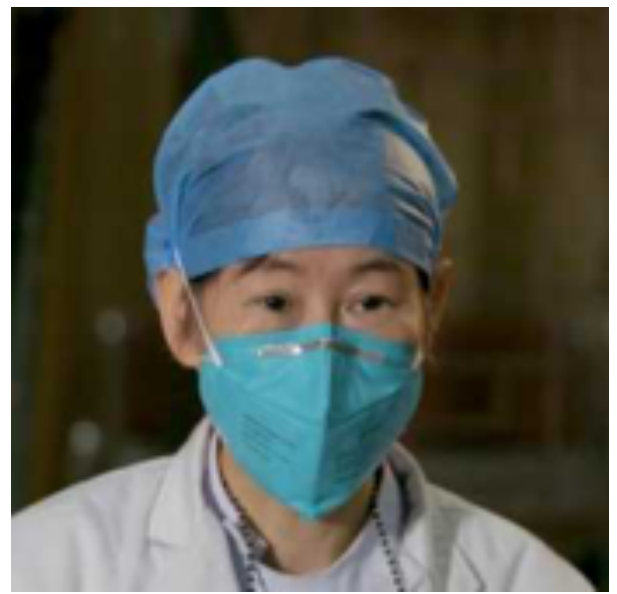

Figura 4. Dra. Zhang Jixian.

de Medicina Tradicional China y Occidental Integrada de Hubei aumentó de unos 100 pacientes diarios a unos 230 pacientes, y cada vez llegaban más casos similares a los iniciales.

Zhang Jixian tenía la experiencia de haber luchado contra el SARS en 2003, cuando tenía 37 años, y fue miembro del grupo de expertos del distrito de Jianghan cuya tarea diaria era buscar a los casos sospechosos en los hospitales.

El 6 de febrero, el día anterior de la muerte de Li Wenliang, el gobierno de Hubei premió y destacó la labor de Zhang Jixian por reconocer una nueva enfermedad por coronavirus y alertar a las autoridades de su institución.

Zhang Jixian tiene el reconocimiento oficial de ser la primera en alertar sobre la nueva enfermedad por coronavirus y es considerada ahora una heroína en China.(20)

\section{Colofón}

Volviendo a nuestros personajes, podemos afirmar que ellos hicieron lo correcto. Ante una desconocida enfermedad potencialmente peligrosa por su letalidad y su rápida diseminación, la obligación de los médicos en cualquier parte del mundo es alertar a sus autoridades inmediatas y a los colegas para que tomen las medidas necesarias.

China es un país con un gobierno autoritario y ejercido por un régimen comunista donde el peso político de la información es muy importante, o más importante, tal que se prefirió ocultar, demorar, posponer la posición de que se estaba enfrentando a un brote epidémico de una enfermedad que rápidamente asociaron con el SARS dada la experiencia que tuvieron en 2003 y que estarían esperando que ocurra de nuevo en cualquier momento.

Por otro lado, los funcionarios chinos reconocieron muy tarde que la nueva enfermedad se transmitía de humano a humano. Por lo menos transcurrieron tres semanas para que se percataran de ello. Zhang Jixian tuvo una ventaja sobre Ai Fen y Li Wenliang, había luchado antes contra el SARS así que pudo reconocer que esta nueva enfermedad tenía un cuadro clínico muy parecido al SARS y, por tanto, que era potencialmente peligrosa para los humanos. 
Pero, siempre hay una historia oficial. Ai Fen también comunicó a las autoridades y no tuvo la respuesta que recibió Zhang Jixian. ¿Acaso porque ella, poco antes o casi simultáneamente, había informado a los colegas? ¿Esto hizo que entrara en descrédito? ¿Existe un vínculo de Zhang Jixian con el Partido Comunista de China que Ai Fen no lo tiene? ${ }^{10}$

Ai Fen, Li Wenlinag y Zhang Jixian tuvieron sus experiencias en paralelo, aunque la última trabajaba en otro hospital. Además, el nexo de Zhag Jixian con sus superiores fue más estrecho que en el caso de Ai Fen, a pesar de que ambas detentaban el mismo cargo en sus hospitales. Lo concreto es que Ai Fen recibió una llamada de atención, Li Wenliang fue amonestado, y vindicado después, y Zhang Jixian es considerada, oficialmente por el gobierno chino, como la primera doctora que comunicó la aparición de una nueva enfermedad contagiosa conocida ahora como el COVID-19.

Quizás, la pandemia hubiera tenido un curso diferente si las respectivas autoridades sanitarias hubieran reaccionado adecuadamente ante el real hecho de estar asistiendo al nacimiento de una potencial epidemia antes que pensar en el supuesto malestar social, aunque también es previsible ver que las implicancias políticas y económicas jugaron un papel importante en tomar las decisiones. Igual podríamos inferir del proceder de la OMS que tardó en medir el potencial de la naciente amenaza a la salud mundial.

Lo sucedido con Ai Fen y Li Wenliang quedará en la memoria de los que vivimos en tiempo real esta pandemia y para los que escribirán la historia en el futuro. Esto no significa menoscabar la contribución oportuna de Zhang Jixian.

\section{REFERENCIAS BIBLIOGRÁFICAS}

1. Leung, Hillary. 'An Eternal Hero.' Whistleblower Doctor Who Sounded Alarm on Coronavirus Dies in China. Time. February 7, 2020. https://time.com/5779678/li-wenliang-coronaviruschina-doctor-death/

2. Davidson H. First Covid-19 case happened in November, China government records show - report. The Guardian. March $13^{\text {th }}, 2020$. https://www.theguardian.com/world/2020/mar/13/first-covid-19case-happened-in-november-china-government-records-showreport

3. Lee Myers, Steve. China Created a Fail-Safe System to Track Contagions. It Failed. https://www.nytimes.com/2020/03/29/world/ asia/coronavirus-china.html

4. CDC. CDC SARS Response Timeline. URL disponible en: https:// www.cdc.gov/about/history/sars/timeline.htm

5. Bik EM. Dr. Ai Fen, 艾芬, the Wuhan Whistle. Science Integrity Digest. March 11, 2020. https://scienceintegritydigest. com/2020/03/11/dr-ai-fen-the-wuhan-whistle/

6. Green, Andrew. Li Wenliang. The Lancet. February 18, 2020. URL disponible en: https://www.thelancet.com/journals/lancet/ article/PIIS0140-6736(20)30382-2/fulltext. doi.org/10.1016/s01406736(20)30382-2

10 Curiosamente, el fotocheck de trabajo de Zhang Jixian indica su filiación comunista.
7. Woodward, Aylin. Several young doctors in China have died of the coronavirus. Medical workers are far more vulnerable to infection than the general population. Mar 3, 2020. URL disponible en: https://www.businessinsider.com/why-coronavirus-killedyoung-chinese-doctors-2020-2

8. OPS. Actualización Epidemiológica. Nuevo coronavirus (Covid-19). 28 de febrero del 2020. URL disponible en: https://www.paho.org/ sites/default/files/2020-02/2020-feb-28-phe-actualizacion-epicovid19.pdf

9. Li, Jane. Why a Chinese virology lab is unable to quell the coronavirus conspiracy theories around it. Quartz. February 20, 2020. URL disponible en: https://qz.com/1805422/wuhan-virologylab-unable-to-quell-china-coronavirus-conspiracies/

10. Bryner, Jeannna. Wuhan lab says there's no way coronavirus originated there. Here's the science. Live Science. April 18, 2020. URL disponible en: https://www.livescience.com/coronaviruswuhan-lab-complicated-origins.html

11. Agencia AFP. Gestión. El laboratorio de Wuhan, centro de una controversia mundial. Abril 18,2020. URL disponible en: https:// gestion.pe/mundo/el-laboratorio-de-wuhan-centro-de-unacontroversia-mundial-coronavirus-covid-19-noticia/?ref=gesr

12. Huang, Joyce. Where is investigation report into coronavirus whistleblower? Voa News on Iran. March 9, 2020. URL disponible en: https://www.voanews.com/science-health/coronavirus-outbreak/ where-investigation-report-coronavirus-whistleblower

13. Plataforma Digital Única del Estado Peruano. Coronavirus en el Perú: casos confirmados. URL disponible en: https://www.gob. pe/8662-coronavirus-en-el-peru

14. Rudolph, Josh. Netizen creativity preserves censored interview with Wuhan doctor. China Digital Times. Mar 11, 2020. URL disponible en: https://chinadigitaltimes.net/2020/03/netizencreativity-preserves-censored-interview-with-wuhan-doctor/

15. WHO. WHO announces COVID-19 outbreak a pandemic. March 12, 2020. URL disponible en: https://www.euro.who.int/en/healthtopics/health-emergencies/coronavirus-covid-19/news/ news/2020/3/who-announces-covid-19-outbreak-a-pandemic

16. Cerveliera, Bernardo. Wuhan Public Security Bureau apologises to Li Wenliang's family over 'inappropriate reprimand'. AsiaNews. It. March 20, 2020. URL disponible en: http://www.asianews.it/ news-en/Wuhan-Public-Security-Bureau-apologises-to-LiWenliang's-family-over-inappropriate-reprimand-49616.html

17. Explained News. Explained: Harvard study indicates Covid-19 may have been circulating in Wuhan since last August. The Indian Express. June 19, 2020. URL disponible en: https://indianexpress. com/article/explained/19study-covid-19-wuhan-august-lastyear-6452538/

18. Wuhan Evening News. How did she find this pneumonia different when she first reported the epidemic? February 2, 2020. URL disponible en: https://news.sina.com.cn/c/2020-02-02/dociimxyqvy9611122.shtml

19. Colville, Alex. Zhong Nanshan, the doctor who led China through COVID-19 and SARS. Chinese Lives. June 22,2020. URL disponible en: https://supchina.com/2020/06/22/zhong-nanshandoctor-who-led-china-through-covid-19-and-sars/

20. Medical worker reports coronavirus. Beijing Review. February 13, 2020. URL disponible en: http://www.bjreview.com/Nation/202002/ t20200210 800192452.html

\section{CORRESPONDENCIA:}

Oscar G. Pamo-Reyna

oscar.pamo@upch.pe

Fecha de recepción: 01-08-2020.

Fecha de aceptación: 17-08-2020.

CONFLICTO DE INTERÉS: Ninguno, según el autor. FINANCIAMIENTO: Ninguno. 